\title{
Effect of intravitreal ranibizumab on serous retinal detachment in branch retinal vein occlusion
}

This article was published in the following Dove Press journal:

Clinical Ophthalmology

\author{
Emine Dogan' \\ Ozkan Sever ${ }^{2}$ \\ Burcin Köklü Çakır' \\ Erkan Celik' \\ 'Department of Ophthalmology, \\ Sakarya University Medical \\ Education and Research Hospital, \\ Sakarya, Turkey; ${ }^{2}$ Department of \\ Ophthalmology, Namik Kemal \\ University School of Medicine, Namik \\ Kemal University, Tekirdag, Turkey
}

Correspondence: Ozkan Sever Department of Ophthalmology, Namik Kemal University School of Medicine, Namik Kemal University, Tekirdag 59000, Turkey

Email sever_ozkan@hotmail.com

\begin{abstract}
Purpose: The aim of this study was to evaluate the effect of initial intravitreal ranibizumab injection on visual acuity (VA) and central macular thickness (CMT) for the treatment of macular edema (ME) with and without serous retinal detachment (SRD) secondary to branch retinal vein occlusion (BRVO).
\end{abstract}

Materials and methods: Fifty-two BRVO eyes, treated with intravitreal ranibizumab injection for ME with and without SRD, were retrospectively reviewed. Patients were divided into two groups according to spectral domain optical coherence tomography (SD-OCT). The efficacy of intravitreal ranibizumab injection at first month was assessed by analyzing the change in best-corrected VA and reduction in CMT with SD-OCT.

Results: There were 21 patients with SRD and 31 patients with only CME (no-SRD). CMT was significantly greater in the SRD group than in the CME group $(451 \pm 62.2 \mu \mathrm{m}$ vs $383.5 \pm 37.2 \mu \mathrm{m}$, respectively, $P<0.05$ ). After initial intravitreal ranibizumab injection, mean VA improved from $0.87 \pm 0.26$ logarithm of the minimum angle of resolution (LogMAR) to $0.54 \pm 0.27 \log$ MAR $(P<0.01)$ and CMT decreased from $451 \pm 62.2 \mu \mathrm{m}$ to $379.3 \pm 58.6 \mu \mathrm{m}(P<0.001)$ in the SRD group. In the no-SRD group, mean VA improved from 0.69 \pm 0.25 LogMAR to $0.44 \pm 0.25$ $\operatorname{LogMAR}(P<0.001)$ and the CMT decreased from $383.5 \pm 37.2 \mu \mathrm{m}$ to $337.7 \pm 39.4 \mu \mathrm{m}(P<0.001)$ at the first month visit. Eyes with SRD revealed better anatomic results and greater reduction of CMT after intravitreal ranibizumab injection $(P<0.01)$.

Conclusion: VA and CMT can be improved by intravitreal ranibizumab injection in BRVO patients with and without SRD. However, more marked improvement in macular morphology was achieved in patients with SRD than those without SRD.

Keywords: ranibizumab, vein occlusion, serous detachment

\section{Introduction}

Branch retinal vein occlusion (BRVO) is the second most common retinal vascular disorder after diabetic retinopathy. ${ }^{1}$ Macular edema (ME), occurring in about $60 \%$ of cases, is the most frequent cause of visual loss in patients with BRVO. ${ }^{2}$ BRVO can lead to fluid leakage from vessels to tissue, which occurs in response to increased intravascular pressure behind the occlusion. In patients with BRVO, retinal ischemia induces the secretion of inflammatory mediators such as vascular endothelial growth factor (VEGF), which is the major cause of breakdown of the blood-retinal barrier, endothelial dysfunction, and increased vascular permeability. ${ }^{3}$

Several treatment modalities including laser photocoagulation, intravitreal dexamethasone implants, and anti-VEGF agents such as ranibizumab, bevacizumab, and aflibercept are currently available for the treatment of ME secondary to BRVO ${ }^{4}$ More recently, anti-VEGF agents have become the most frequently used therapeutics for ME 
in BRVO. VEGF and the aqueous concentration of inflammatory factors have been found significantly elevated in eyes with ME secondary to BRVO. ${ }^{5}$ Anti-VEGF agents can achieve anatomical resolution of $\mathrm{ME}$, stabilization, and improvement of the best-corrected visual acuity (VA) in BRVO patients. ${ }^{6,7}$ Ranibizumab (Lucentis, Novartis AG, Basel, Switzerland) is the first VEGF inhibitor to be US Food and Drug Administration approved for use in BRVO, and several randomized prospective trials showed its efficacy. ${ }^{8}$ Both the BRAVO and A Study of the Efficacy and Safety of Ranibizumab Injection in Patients With Macular Edema Secondary to Central Retinal Vein Occlusion (CRUISE) trials demonstrated the benefits of ranibizumab on VA and central macular thickness (CMT) in patients with BRVO and central retinal vein occlusion. ${ }^{8,9}$

Optical coherence tomography (OCT) studies have revealed that ME secondary to BRVO is frequently associated with cystoid macular edema (CME), serous retinal detachment (SRD), and inner retinal thickening. SRD, which is associated with several ischemic, inflammatory, and neoplastic diseases, has been reported in $15 \%-80 \%$ of patients with BRVO., ${ }^{2,10,11}$ Although the mechanism of SRD is not completely understood, extensive leakage from retinal circulation into the subretinal space exceeding its drainage capacity may lead to accumulation in the subretinal space and cause serous detachment. ${ }^{12}$ Also, pathogenesis of SRD can be related to inflammation that enhances vascular permeability and dysfunction in various retinal structures. Recently, studies showed that SRD and loss of the photoreceptor layer damage the retinal pigment epithelium and neurosensory retina leading to a poor visual prognosis in BRVO. ${ }^{13-15}$ However, there are a limited number of studies that assess treatment outcomes in eyes with SRD in BRVO.

The aim of this study was to evaluate the effect of initial intravitreal ranibizumab injection on VA and CMT for the treatment of $\mathrm{ME}$ in eyes with and without SRD secondary to BRVO.

\section{Materials and methods}

We retrospectively reviewed the medical records of patients, who had ME secondary to BRVO and were treated with intravitreal ranibizumab injection, examined at the Department of Ophthalmology, Sakarya University Medical Education and Research Hospital, and Namık Kemal University, Department of Ophthalmology, from February 2014 to January 2016. This study was performed in accordance with the Declaration of Helsinki. Prior approval to review the patient data was taken from the hospital directories
(February 2016). Written informed consent was taken from all the patients. Because this is a retrospective study, there is no need for institutional reviewer board approval in accordance with the rules declared by the Ministry of Health of Turkey.

Exclusion criteria included prior ocular surgery, retinal, optic disc or iris neovascularization, diabetic retinopathy, previous laser photocoagulation and/or intravitreal injection, current use of systemic steroids and/or immunomodulators, clinically significant media opacity, presence of vitreomacular traction detected by OCT, history of ocular inflammation, and marked retinal hemorrhage (including macular bleeding involving the fovea).

On initial examination, patients underwent complete ophthalmologic examination, including VA measurement, slitlamp biomicroscopy, fundoscopy, fundus color photography, fluorescein angiography, and spectral domain OCT (Cirrus, version 6.5.0, Carl Zeiss Meditec Inc, Dublin, CA) analysis $(512 \times 128$ macular cube acquisition protocol) to measure the CMT and evaluate the absence or presence of CME/SRD.

Best-corrected VA was measured by Snellen chart and then converted into logarithm of the minimum angle of resolution (LogMAR) for statistical comparison. OCT images were obtained from each eye following pupil dilation by the same operator. The retinal thickness was defined as the distance between the inner surface and the outer surface of retina. CME was defined as hyporeflective intraretinal cavities in cross-sectional scans radiating from the center of the macula. In contrast, SRD was defined as the presence of nonreflective cavity with minimal shadowing of the underlying tissues due to subretinal fluid accumulation leading to detachment of the neurosensory retina. Patients were divided into two groups according to spectral domain OCT findings of SRD and CME: patients with only CME (Group No-SRD) and patients with SRD (Group $\mathrm{SRD})$. All the eyes in the SRD group also had CME.

The BRVO eye was anesthetized with topical anesthetics and prepared according to standard clinical practice for eyes undergoing intravitreal injection. Intravitreal injection of $0.5 \mathrm{mg} / 0.05 \mathrm{~mL}$ ranibizumab was performed under sterile conditions with a 30-gauge needle. Immediately after the injection, ciprofloxacin ointment was given for 1 week. After the initial injection of ranibizumab, patients were followed up on the first day, third day, and seventh day and 1-month intervals with VA measurement and OCT analysis with the $512 \times 128$ macular cube acquisition protocol of the Cirrus HD OCT. This study investigated the first month's results of initial intravitreal ranibizumab injection. 
Statistical analysis was performed with Python version 2.7.6 and SciPy library version 0.13.3. Results are presented as mean \pm SD. Welch's test was used to compare normally distributed unpaired continuous variables between the groups. Two-tailed $P$-values of $<0.05$ were considered to indicate statistical significance.

\section{Results}

A total of 52 eyes of 52 patients met the inclusion criteria and were enrolled in the study. The mean patient age was $64.9 \pm 4.9$ years (range, 53-76 years). Each patient had unilateral BRVO and ME. The patients were divided into two groups according to the presence of SRD. There were 21 patients with SRD and 31 patients with only CME (no-SRD). All the 21 patients in the SRD group had both SRD and CME. There were no differences between groups with regard to gender, age, laterality, and duration of BRVO. Table 1 describes the demographic characteristics of the study population.

At baseline, VA was significantly worse in the SRD group than in the no-SRD group $(0.87 \pm 0.26$ vs $0.69 \pm 0.25$, respectively, $P<0.05)$. Also, CMT was significantly greater in the SRD group than in the CME group $(451 \pm 62.2 \mu$ vs $383.5 \pm 37.2 \mu$, respectively, $P<0.05$ ).

In the analysis of each group, we found significant improvement in VA and reduction in CMT between the baseline and the final follow-up visits after intravitreal injection of ranibizumab. In the SRD group, mean VA improved from $0.87 \pm 0.26 \operatorname{LogMAR}$ at baseline to $0.54 \pm 0.27 \mathrm{LogMAR}$ $(P<0.001)$ at the final visit. The CMT decreased from $451 \pm 62.2 \mu \mathrm{m}$ to $379.3 \pm 58.6 \mu \mathrm{m}(P<0.001)$. In the no-SRD group, mean VA improved from $0.69 \pm 0.25 \operatorname{LogMAR}$ at baseline to $0.44 \pm 0.25 \operatorname{LogMAR}(P<0.001)$ at the first month visit, and the CMT decreased from $383.5 \pm 37.2 \mu \mathrm{m}$ to $337.7 \pm 39.4 \mu \mathrm{m}(P<0.001)$. The final VA and CMT parameters were better in patients with SRD group after initial

Table I Clinical characteristics of the patients at baseline

\begin{tabular}{llll}
\hline & SRD & No-SRD & $P$-value \\
\hline Gender (M/F), n & 21 & 31 & 0.78 \\
Age $(y)$, mean \pm SD & $64.7 \pm 4.9$ & $65.4 \pm 4.6$ & 0.58 \\
Right eye/left eye, $\mathrm{n}$ & $13 / 8$ & $17 / 14$ & $0.6 \mathrm{I}$ \\
Superior/inferior temporal, $\mathrm{n}$ & $14 / 7$ & $21 / 10$ & 0.20 \\
Duration of BRVO (months), & $2.3 \pm 2.1$ & $2.7 \pm 1.9$ & 0.63 \\
mean \pm SD & & & \\
Mean CMT ( $\mu$ m, mean \pm SD & $451 \pm 62.2$ & $383.5 \pm 37.2$ & $<0.05$ \\
Mean VA (LogMAR), & $0.87 \pm 0.26$ & $0.69 \pm 0.25$ & $<0.05$ \\
mean \pm SD & & &
\end{tabular}

Abbreviations: BRVO, branch retinal vein occlusion; CMT, central macular thickness; $F$, female; LogMAR, logarithm of the minimum angle of resolution; $M$, male; SRD, serous retinal detachment; VA, visual acuity.

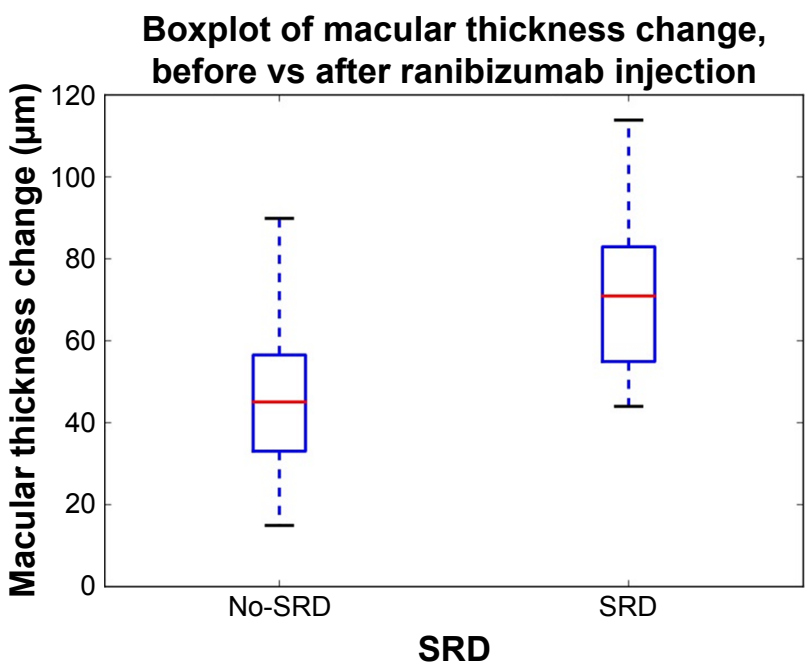

Figure I First month macular thickness changes of the groups with serous retinal detachment (SRD) and without SRD after single-dose intravitreal ranibizumab injection.

intravitreal ranibizumab injection $(P=0.13$ and $P<0.01$, respectively) (Figures 1 and 2).

\section{Conclusion}

In the present study, we investigated the prognostic value of SRD on VA and CMT after first intravitreal ranibizumab injection in BRVO patients with ME. Before intravitreal injection, the mean VA was significantly worse in the SRD group than in the CME group and eyes with SRD had greater CMT. A significant improvement of the VA and resolution

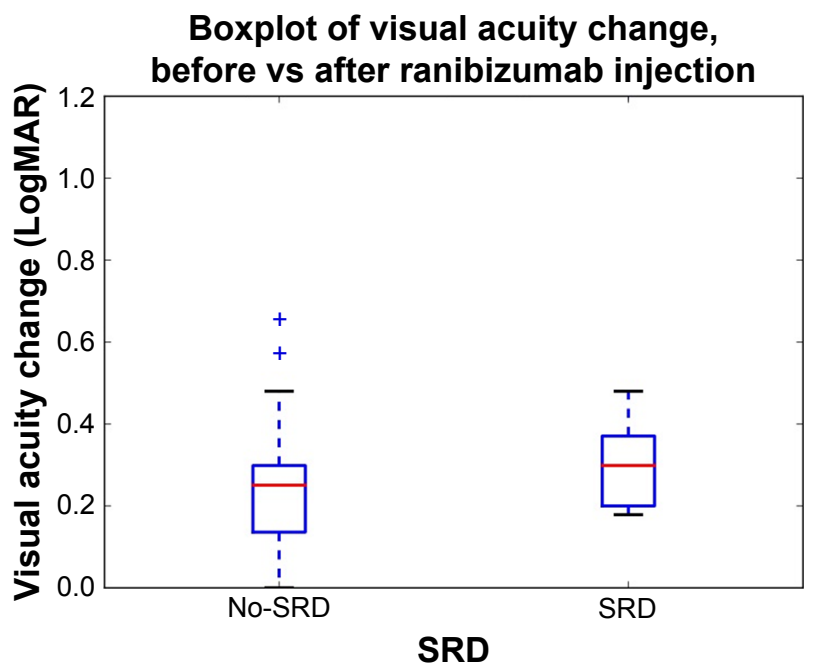

Figure 2 First month visual acuity changes of the groups with serous retinal detachment (SRD) and without SRD after single-dose intravitreal ranibizumab injection. 
of the SRD were found after the initial injection of ranibizumab in both groups. However, eyes with SRD revealed better anatomic results and greater reduction of CMT after intravitreal ranibizumab injection.

$\mathrm{ME}$ is a leading cause of vision loss in BRVO, which can be associated with various morphological changes such as sponge-like retinal swelling, cystic changes, and SRD. ${ }^{16}$ In SRD, fluid accumulates between the neurosensory retina and the retinal pigment epithelium, and these areas are seen as hyporeflective spaces on OCT. A variety of mechanisms have been suggested for the occurrence of SRD. It was previously thought that SRD pathogenesis was associated with hemodynamic overload and disruption in the function of the retinal pigment epithelium due to retinal ischemia. However, retinal pigment epithelium barrier seems to be resistant to acute ischemia, releasing proinflammatory mediators; VEGF-mediated enhanced permeability can cause cytoskeletal stress and retinal pigment epithelium barrier dysfunction. ${ }^{17,18}$ In some studies, inflammatory factors were found to be related to the pathogenesis of SRD. Dacheva et al reported that inflammatory cytokines were found to be correlated with CMT and the extent of SRD. ${ }^{19}$ Noma et al found that vitreous fluid levels of VEGF and soluble intercellular adhesion molecule-1 were higher in BRVO patients with SRD compared with those with CME. They reported that VA was significantly worse in the SRD group than in the CME group, and CMT was significantly greater in the SRD group similar to our study. ${ }^{5}$ They assumed that worse VA in the SRD group may be related to photoreceptor cell damage caused by macular detachment and functional impairment secondary to ischemia with high vitreous fluid levels of VEGF. ${ }^{5}$

Another possible mechanism suggested for the development of SRD is vitreal hyperosmolarity which flows the fluid from the vitreous to the choroid. ${ }^{20,21}$ Ota et al declared that also small breaks on the external surface of the neurosensory retina, which connects the swollen outer reina, could be responsible for SRD. ${ }^{10}$ Tsujikawa et al reported that Müller cell cone traction due to formation of foveal cystoid spaces would lead to traction on the inner and outer segments of the foveal photoreceptors and loss of the barrier function of the external limiting membrane, which will cause diffusion of intraretinal fluid into the subretinal space and increase of SRD. ${ }^{13}$ Formation of SRD causes damage to photoreceptors in the outer segment and impairment of VA.

Various intravitreal pharmacotherapies have been tried for the treatment of CME secondary to BRVO, such as ranibizumab, bevacizumab, aflibercept, triamcinolone acetonide, and dexamethasone implant. ${ }^{6,14,22}$ Karacorlu et al investigated the efficacy of intravitreal triamcinolone acetonide for CME with SRD in BRVO, and they reported improved vision and significant decrease of the CMT after intravitreal triamcinolone acetonide treatment. ${ }^{14}$ Triamcinolone acetonide was thought to reduce CMT by decreasing retinal capillary permeability via an effect on tight junctions. ${ }^{6}$ In addition, decrease in retinal capillary permeability may relieve traction on the Müller cell cone, which may lead to reduction in SRD. It was also reported that corticosteroids may prevent the production of various inflammatory molecules that promote leukocyte adhesion and breakdown of the blood-retinal barrier. ${ }^{6}$

VEGF inhibitors have a favorable safety profile and have been widely used for the treatment of ME secondary to BRVO. ${ }^{23-25}$ Cinal et al reported a significant improvement in the vision and resolution of the SRD after bevacizumab treatment for central retinal vein occlusion with SRD. ${ }^{22}$ GallegoPinazo et al analyzed the impact of SRD in the visual prognosis after repeated intravitreal ranibizumab treatment, and they reported that the presence of SRD may be a baseline predictive factor for ranibizumab treatment outcomes in BRVO patients, with no influence on the number of treatments needed between patients with or without SRD at baseline. ${ }^{26}$ They also emphasized that the patients with SRD showed both poorer vision and greater CMT at baseline, similar to our study, and the presence of SRD is related to a worse visual outcome. ${ }^{26}$

Retinal vein occlusion pathogenesis involves a cascade of immune and inflammatory changes. ${ }^{2}$ Pfister et al assessed the levels of inflammatory and angiogenic cytokines in untreated BRVO patients with ME and correlated the results with OCT parameters. They found that VEGF-A was more often correlated with the morphologic changes such as SRD. ${ }^{27}$ In a similar study, investigating the correlation from undiluted vitreous cytokines of untreated central retinal vein occlusion with OCT, it was reported that inflammatory cytokines, IL-6 and monocyte chemoattractant protein-1 (MCP-1), were more often correlated with predictive morphologic changes such as SRD, which are clinically important in VA prognosis. ${ }^{28}$ Shchuko et al studied the concentrations of intraocular cytokines in 44 BRVO patients before and after intravitreal ranibizumab injection and compared the results with treatment efficacy. They observed that certain angiogenic (VEGF) and proinflammatory (IL-6, IL-8, IL-13, IL-15, MCP-1) factors appeared to be significantly suppressed after ranibizumab injection, and clinical efficacy of the therapy correlated with the degree of cytokines supression. ${ }^{29}$ Funk et al also demonstrated that anti-VEGF monotherapy has an impact on the expression of VEGF and on other inflammatory 
markers. ${ }^{30}$ Better anatomic outcomes after intravitreal ranibizumab injection in the SRD group of our study may be explained by these factors.

The involvement of cytokines is also important in the development and progression of diabetic ME. Kim et al reported that aqueous concentrations of cytokines varied according to the morphologic pattern of diabetic ME, higher in SRD, which might explain the variable response to treatments such as anti-VEGFs or triamcinolone injection. ${ }^{31}$ Koytak et al compared the effect of a single intravitreal bevacizumab injection in eyes with diabetic ME of different patterns determined by OCT, and they found that SRD and CME subtypes were associated with a greater reduction in CMT. However, the change in VA in response to bevacizumab was not significantly different among groups. ${ }^{32}$

In this retrospective study, our results suggest that VA and CMT can be improved by intravitreal ranibizumab injection in BRVO patients with and without SRD. However, more marked improvement of macular morphology was achieved in patients with SRD than those without SRD. This may be explained by the strong association of the inflammatory factors and extensive morphological changes with the occurrence of SRD, thus revealing better anatomic outcomes.

The limitations of this study were its retrospective nature, assessment of single injection results, short follow-up time, a small sample size, lack of investigation of BRVO patients with only SRD, lack of the ratio or actual amount of CME in SRD group, lack of functional mapping by microperimetry (macular sensitivity), and long-term results of these eyes. Identification of characteristics that influence the treatment response to ranibizumab would help to build up more effective management algorithms for different subtypes of ME secondary to BRVO. This study is not sufficient to reach definite conclusions. Randomized, controlled, and comparative trials, with a greater number of eyes and a longer follow-up time, are needed to confirm the role of SRD as a predictive factor in BRVO eyes with ME treated with ranibizumab.

\section{Acknowledgments}

This study was presented at the 14th European School for Advanced Studies in Ophthalmology Retina Academy, Istanbul, November 13-15, 2014, and an abstract of this study has been published in Ophthalmologica. 2014;232:91.

\section{Disclosure}

The authors have no financial or proprietary interest in any product, method, or material described herein, and report no conflicts of interest in this work.

\section{References}

1. Yamaguchi Y, Otani T, Kishi S. Serous macular detachment in branch retinal vein occlusion. Retina. 2006;26(9):1029-1033.

2. Sekiryu T, Iida T, Sakai E, et al. Fundus autofluorescence and optical coherence tomography findings in branch retinal vein occlusion. J Ophthalmol. 2012;2012:1-8.

3. Channa R, Smith M, Campochiaro PA. Treatment of macular edema due to retinal vein occlusions. Clin Ophthalmol. 2011;5:705-713.

4. Glanville J, Patterson J, Mccool R, Ferreira A, Gairy K, Pearce I. Efficacy and safety of widely used treatments for macular oedema secondary to retinal vein occlusion: a systematic review. BMC Ophthalmol.2014; 14(14):7.

5. Noma H, Funatsu H, Mimura T, Tatsugawa M, Shimada K, Eguchi S. Vitreous inflammatory factors and serous macular detachment in branch retinal vein occlusion. Retina. 2012;32(1):86-91.

6. Noma H, Funatsu H, Mimura T, Shimada K. Comparison of the efficacy of intravitreal triamcinolone acetonide for cystoid macular edema with versus without serous retinal detachment in branch retinal vein occlusion: influence on macular sensitivity and morphology. $B M C$ Ophthalmol. 2012;12(1):1-10.

7. Brown DM, Campochiaro PA, Bhisitkul RB, et al. Sustained benefits from ranibizumab for macular edema following branch retinal vein occlusion: 12-month outcomes of a phase III study. Ophthalmology. 2011;118(8):1594-1602.

8. Brown DM, Campochiaro PA, Singh RP, et al. Ranibizumab for macular edema following central retinal vein occlusion: six-month primary end point results of a phase III study. Ophthalmology. 2010;117(6): 1124-1133.

9. Campochiaro PA, Heier JS, Feiner L, et al. Ranibizumab for macular edema following branch retinal vein occlusion: six-month primary end point results of a phase III study. Ophthalmology. 2010;117(6): $1102-1112$

10. Ota T, Tsujikawa A, Murakami T, et al. Subfoveal serous retinal detachment associated with extramacular branch retinal vein occlusion. Clin Ophthalmol. 2013;7:237-241.

11. Keane PA, Sadda SR. Predicting visual outcomes for macular disease using optical coherence tomography. Saudi J Ophthalmol. 2011; 25(2): $145-158$.

12. Otani T, Yamaguchi Y, Kishi S. Movement of intraretinal fluid from distant branch retinal vein occlusion to the submacular space. Clin Ophthalmol. 2013;7:81-86.

13. Tsujikawa A, Sakamoto A, Ota M, et al. Serous retinal detachment associated with retinal vein occlusion. Am J Ophthalmol. 2010;149(2): 291-301.

14. Karacorlu M, Ozdemir H, Karacorlu SA. Resolution of serous macular detachment after intravitreal triamcinolone acetonide treatment of patients with branch retinal vein occlusion. Retina. 2005; 25(7):856-860.

15. Noma H, Funatsu H, Mimura T, Shimada K. Visual function and serous retinal detachment in patients with branch retinal vein occlusion and macular edema: a case series. BMC Ophthalmol. 2011;11:29.

16. Battaglia Parodi M, Isola V. Branch retinal vein occlusion and exudative retinal detachment: pathogenetical aspects. Ophthalmologica. 1994;208(1):29-31.

17. Spaide RF, Lee JK, Klancnik JK, Gross NE. Optical coherence tomography of branch retinal vein occlusion. Retina. 2003;23(3):343-347.

18. Daruich A, Matet A, Moulin A, et al. Mechanisms of macular edema: Beyond the surface. Prog Retin Eye Res. 2018;63:20-68.

19. Dacheva I, Ceglowska K, Nobl M, et al. Correlation from undiluted vitreous cytokines of untreated central retinal vein occlusion with spectral domain optical coherence tomography. Klin Monbl Augenheilkd. 2016;233(7):864-868.

20. Murakami T, Tsujikawa A, Miyamoto K, et al. Relationship between perifoveal capillaries and pathomorphology in macular oedema associated with branch retinal vein occlusion. Eye. 2012;26(6): 771-780. 
21. Marmor MF. Control of subretinal fluid: experimental and clinical studies. Eye (Lond). 1990;4(Pt 2):340-344.

22. Cinal A, Ziemssen F, Bartz-Schmidt KU, Gelisken F. Intravitreal bevacizumab for treatment of serous macular detachment in central retinal vein occlusion. Graefes Arch Clin Exp Ophthalmol. 2011;249(4): 513-520.

23. Campochiaro PA, Brown DM, Awh CC, et al. Sustained benefits from ranibizumab for macular edema following central retinal vein occlusion: twelve-month outcomes of a phase III study. Ophthalmology. 2011;118(10):2041-2049.

24. Spaide RF, Chang LK, Klancnik JM, et al. Prospective study of intravitreal ranibizumab as a treatment for decreased visual acuity secondary to central retinal vein occlusion. Am J Ophthalmol. 2009; 147(2):298-306.

25. Pieramici DJ, Rabena M, Castellarin AA, et al. Ranibizumab for the treatment of macular edema associated with perfused central retinal vein occlusions. Ophthalmology. 2008;115(10):e47-e54.

26. Gallego-Pinazo R, Dolz-Marco R, Pardo-López D, et al. Ranibizumab for serous macular detachment in branch retinal vein occlusions. Graefes Arch Clin Exp Ophthalmol. 2013;251(1):9-14.

27. Pfister M, Rothweiler F, Michaelis M, et al. Correlation of inflammatory and proangiogenic cytokines from undiluted vitreous samples with spectral domain OCT scans, in untreated branch retinal vein occlusion. Clin Ophthalmol. 2013;7:1061-1067.
28. Koss M, Pfister M, Rothweiler F, et al. Correlation from undiluted vitreous cytokines of untreated central retinal vein occlusion with spectral domain optical coherence tomography. Open Ophthalmol J. 2013; 7:11-17.

29. Shchuko AG, Zlobin IV, Yur'eva TN, Ostanin AA, Chernykh ER. Intraocular cytokines imbalance in retinal vein occlusion and its impact on the efficacy of anti-angiogenic therapy. Vestn Oftalmol. 2015; 131(2):50-56.

30. Funk M, Kriechbaum K, Prager F, et al. Intraocular concentrations of growth factors and cytokines in retinal vein occlusion and the effect of therapy with bevacizumab. Invest Ophthalmol Vis Sci. 2009;50(3): $1025-1032$.

31. Kim M, Kim Y, Lee SJ. Comparison of aqueous concentrations of angiogenic and inflammatory cytokines based on optical coherence tomography patterns of diabetic macular edema. Indian J Ophthalmol. 2015;63(4):312-317.

32. Koytak A, Altinisik M, Sogutlu Sari E, Sari ES, et al. Effect of a single intravitreal bevacizumab injection on different optical coherence tomographic patterns of diabetic macular oedema. Eye. 2013; 27(6):716-721.
Clinical Ophthalmology

\section{Publish your work in this journal}

Clinical Ophthalmology is an international, peer-reviewed journal covering all subspecialties within ophthalmology. Key topics include: Optometry; Visual science; Pharmacology and drug therapy in eye diseases; Basic Sciences; Primary and Secondary eye care; Patient Safety and Quality of Care Improvements. This journal is indexed on

\section{Dovepress}

PubMed Central and CAS, and is the official journal of The Society of Clinical Ophthalmology (SCO). The manuscript management system is completely online and includes a very quick and fair peer-review system, which is all easy to use. Visit http://www.dovepress.com/ testimonials.php to read real quotes from published authors. 